\title{
THE TWO MODES OF GAS GIANT PLANET FORMATION
}

\author{
Aaron C. Boley \\ Institute for Theoretical Physics, University of Zurich, Winterthurerstrasse 190, Zurich, CH-8057, Switzerland; aaron.boley@gmail.com \\ Received 2008 December 3; accepted 2009 March 5; published 2009 March 23
}

\begin{abstract}
I argue for two modes of gas giant planet formation and discuss the conditions under which each mode operates. Gas giant planets at disk radii $r>100$ AU are likely to form in situ by disk instability, while core accretion plus gas capture remains the dominant formation mechanism for $r<100$ AU. During the mass accretion phase, mass loading can push disks toward fragmentation conditions at large $r$. Massive, extended disks can fragment into clumps of a few to tens of Jupiter masses. This is confirmed by radiation hydrodynamics simulations. The two modes of gas giant formation should lead to a bimodal distribution of gas giant semimajor axes. Because core accretion is expected to be less efficient in low-metallicity systems, the ratio of gas giants at large $r$ to planets at small $r$ should increase with decreasing metallicity.
\end{abstract}

Key words: hydrodynamics - instabilities - planetary systems: formation - planetary systems: protoplanetary disks - radiative transfer

\section{INTRODUCTION}

Both core accretion plus gas capture (e.g., Pollack et al. 1996) and direct formation by disk instability (Cameron 1978; Boss 1997) can form gas giant planets in principle. In order for gravitational instabilities (GIs) to form gas giants directly, the Toomre (1964) $Q=c_{s} \kappa / \pi G \Sigma$, where $c_{s}$ is the sound speed, $\kappa$ is the epicyclic frequency, and $\Sigma$ is the surface density, must approach unity and the local cooling time must be $\lesssim$ the local orbit period (Gammie 2001; Rice et al. 2005; Rafikov 2005, 2007). An isothermal, low- $Q$ disk is strongly susceptible to fragmentation (e.g., Tomley et al. 1994; Boss 1998; Nelson et al. 1998; Mayer et al. 2002; Pickett et al. 2003).

Analytical work (e.g., Rafikov 2007) and radiation hydrodynamics simulations (Nelson et al. 2000; Cai et al. 2006; Boley et al. 2006, 2007a; Stamatellos \& Whitworth 2008; Boley \& Durisen 2008) find that disk fragmentation inside $r \sim$ tens of AU is unlikely because regions with high cooling rates and low $Q$ are rare (cf. most recently, Boss 2008 and Mayer et al. 2007). When $Q$ is low, the gas is cool, so cooling times are typically long. When cooling times are short, the gas is hot, so $Q$ is typically high. In contrast, Boss (2004) argues that convection can increase the cooling rates enough to cause fragmentation. Likewise, Mayer et al. (2007) reported convection-induced fragmentation in their simulations when the mean molecular weight is increased from 2.4 to 2.7. ${ }^{1}$ Boley et al. (2006) and Boley $\&$ Durisen (2008) argued in return that the reported convective motions are likely shock bores along the spiral shocks (Boley $\&$ Durisen 2006) or an artifact of suddenly changing the mean molecular weight. Moreover, Rafikov (2007) and Boley et al. (2006, 2007a) found that convection does not decrease cooling times enough to trigger fragmentation because sustained convection is regulated by the entropy gradient and energy must be radiated away at the disk's surface. Core accretion seems to be the only option for gas giant formation inside $r \sim 100 \mathrm{AU}$. However, the recently discovered substellar companions with wide semimajor axes $a \sim 100 \mathrm{AU}$ and masses $M \sim$ few to tens $M_{\mathrm{J}}$ (e.g., Luhman et al. 2006; Lafreniere et al. 2008; Kalas et al. 2008) challenge the interpretation that core accretion is

\footnotetext{
1 Boley et al. (2006) simulated a disk with a $\mu \sim 2.7$; they did not see fragmentation.
}

the sole formation mechanism for gas giants. The susceptibility of disks to fragmentation at large $r$ remains an open question. Stamatellos et al. (2007; hereafter SHW2007) and Stamatellos \& Whitworth (2009; hereafter SW2009) found that extended, massive disks can fragment under realistic conditions (cf. Boss 2006); but, their results are based on running simulations with highly unstable initial conditions, which as argued here, can significantly change the outcome of disk evolution.

Consider a disk where the sound speed becomes constant with radius for large $r$. For $\Sigma \sim r^{-q}, Q \sim r^{q} \Omega \sim r^{q-1.5}$. As long as $q<1.5$, the stabilizing shear contribution to $Q$ falls off faster than the destabilizing self-gravity contribution, and the disk can become susceptible to GIs. Now consider the radiative cooling time for some fluid element $t_{\mathrm{rad}}=\epsilon /|\nabla \cdot F|$, where $\epsilon$ is the internal energy density of the gas. Using the free-streaming limit for the divergence of the flux, where $|\nabla \cdot F|=4 \rho \kappa_{p} \sigma T^{4}$ for low optical depth $\tau=\int_{-\infty}^{\infty} \rho \kappa_{P} d z$, the cooling time can be approximated by $t_{\mathrm{rad}} \sim \Sigma c_{s}^{2} /\left(4 \tau \sigma T^{4}\right)$, where $\rho$ is the mass density at temperature $T$ and $\kappa_{P}$ is the Planck mean opacity. For $\tau \ll 1$, radiative cooling becomes extremely inefficient. Because cooling in the optically thick disk is also inefficient, the $\tau \sim 1$ transition region may be the most conducive regime for disk fragmentation (e.g., Rafikov 2005). The cooling time in a disk with $\Sigma \sim 1 \mathrm{~g} \mathrm{~cm}^{-2}, \tau \sim 0.1$, and $T \sim 10 \mathrm{~K}$, is $t_{\text {rad }} \sim 600 \mathrm{yr}$, which is less than the orbital period around a $1 M_{\odot}$ star at $r \sim 100 \mathrm{AU}$.

What if the $\tau \sim 1$ and $Q \sim 1$ regions do not coincide? Mass loading, i.e., mass accretion onto the disk, is crucial for driving fragmentation at $r>100 \mathrm{AU}$. Numerical fragmentation studies have focused mainly on thermal energy balance arguments. However, there are additional considerations when evaluating the stability of a disk against fragmentation. The rate that $Q$ changes in a local region of a disk is

$$
\frac{d \ln Q}{d t}=\frac{1}{2} \frac{d \ln c_{s}^{2}}{d t}+\frac{d \ln \kappa}{d t}-\frac{d \ln \Sigma}{d t} .
$$

When GIs are in a self-regulating phase, the effective Shakura \& Syunyaev (1973) $\alpha$ tends toward a few hundredths or less (e.g., Gammie 2001; Lodato \& Rice 2004; Mejía et al. 2005; Boley et al. 2006), so the timescale for $\Sigma$ and $\kappa$ to change due to mass transport should be much larger than the local dynamical time. 
For fragmentation criteria studies of isolated disks, the thermal energy term is the most relevant. In order to avoid fragmentation, the local cooling time for the gas $t_{\text {cool }}>f(\gamma) t_{d}$, where $t_{d}$ is the local gas orbital period and $f(\gamma)$ is some function of the adiabatic index of order unity. In the local model formalism, the $t_{\text {cool }}$ criterion is associated with a critical effective $\alpha_{c} \sim 0.06$ (Gammie 2001; Rice et al. 2005). This $\alpha$-limit indicates the point at which local energy dissipation by GIs cannot balance cooling, and as a result, the disk fragments.

In addition to energy transport, $\alpha_{c}$ can be related to a critical mass flux $\dot{M}_{c} \approx 3 \pi \alpha_{c} c_{s}^{2} \Sigma / \Omega$. Substituting $Q$ into this relation gives $\dot{M}_{c} \approx 3 \alpha_{c} c_{s}^{3} /(G Q)$ (see also Goodman 2003; Matzner \& Levin 2005). At large $r$, where the temperature of the disk approaches the envelope temperature, the ratio $\dot{M}_{c} / \dot{M}_{e} \sim 3 \alpha_{c} / Q$, where $\dot{M}_{e} \sim c_{s}^{3} / G$ is the mass accretion onto the disk from the envelope. For an envelope temperature of $T=30 \mathrm{~K}, \dot{M}_{e} \sim 2 \times 10^{-5} M_{\odot} \mathrm{yr}^{-1}$. Because $\alpha_{c} \sim 0.06$, mass loading can operate at multiple times the maximum local transport flux. Episodes of nonlocal transport and/or disk fragmentation should occur for realistic envelope accretion rates.

I present results from radiative hydrodynamics simulations that show mass loading can drive a disk toward fragmentation at large $r$. The fragments form clumps with masses greater than a few $M_{J}$. Throughout this Letter, I refer to the inner disk as the region inside $r \sim 100 \mathrm{AU}$ and the extended disk as the region outside this boundary. This transition radius is illustrative, but represents a reasonable estimate for where fragmentation becomes possible (e.g., Matzner \& Levin 2005).

\section{METHODOLOGY}

I use CHYMERA (Boley 2007) to model the formation of massive extended disks. CHYMERA is an Eulerian code that solves for the equations of hydrodynamics with self-gravity on an evenly spaced, cylindrical grid. The rotational states of molecular hydrogen are taken into account (Boley et al. $2007 \mathrm{~b}$ ), and the radiative transfer scheme has passed a series of analytic tests. The radiative transfer algorithm and test results are presented in Boley et al. (2007a). D'Alessio et al. (2001) opacity tables are used for calculating optical depths, with a maximum grain radius $a_{\max }=1 \mu \mathrm{m}$. In the following simulations, an equilibrium ortho:parahydrogen ratio $(\mathrm{O}: \mathrm{P})$ is used. The gas is assumed to have a mixture of $0.73,0.25$, and 0.02 for hydrogen, helium, and metals, respectively.

Preliminary simulations using the CHYMERA radiative transfer scheme show that the radiative cooling time is shorter than the local orbital time and that the disk is effectively isothermal, in agreement with the estimate in Section 1. For modeling spiral waves, the normal radiative transfer scheme is stable in these simulations because the gas temperature is never far from the incident irradiation temperature. However, for some preliminary simulations, the cooling algorithm became numerically unstable immediately after clump formation due to the sudden increase in temperature, creating highly disparate radiative transfer and hydrodynamics timescales. In order to study fragmentation at large $r$, the following radiative cooling algorithm is used for the simulations presented here. The divergence of the flux

$$
\nabla \cdot F=-(A / V) \sigma\left(T^{4}-T_{\mathrm{irr}}^{4}\right) f_{\tau}^{-1},
$$

where $A / V$ is the cell area-to-volume ratio, $T_{\text {irr }}$ is the incident irradiation on the disk at $r$, and $f_{\tau}=\Delta \tau+1 / \Delta \tau$. The local optical depth across a cell is calculated by $\Delta \tau=\rho\left(\kappa_{\text {Rosseland }}(1-\right.$ $\left.\left.\exp \left[-2 \Delta \tau_{\text {Planck }}\right]\right)+\kappa_{\text {Planck }} \exp \left[-2 \Delta \tau_{\text {Planck }}\right]\right) V^{1 / 3}$, where $\Delta \tau_{\text {Planck }}$ is an initial estimate using the Planck mean opacity. This cooling approximation goes to the free-streaming limit for small $\Delta \tau$ and to zero for large $\Delta \tau$. In order to ensure that the algorithm is stable for large hydrodynamic time steps, the divergence of the flux is adjusted such that

$$
\begin{aligned}
\nabla \cdot F^{\text {adjusted }}= & \nabla \cdot F \exp \left[-\left(\Delta t_{\text {hydo }} / \Delta t_{\text {rad }}\right)^{2}\right]+\rho\left(e_{\text {equil }}-e\right) / \\
& \Delta t_{\text {hydro }}\left(1-\exp \left[-\left(\Delta t_{\text {hydro }} / \Delta t_{\text {rad }}\right)^{2}\right]\right)
\end{aligned}
$$

Here, $e$ is the specific internal energy of the gas, $e_{\text {equil }}$ is the internal energy of the gas if it were at $T_{\text {irr }}, \Delta t_{\text {hydro }}$ is the Courant time step, and $\Delta t_{\mathrm{rad}}=\rho e /|\nabla \cdot F|$ is the radiative timescale. The irradiation temperature for these simulations is set to $30 \mathrm{~K}$ for all $r$.

The central protostar's position is integrated self-consistently with a softened potential, where $\Phi_{\text {star }}=G M_{\text {star }} /\left(\left|\mathbf{r}-\mathbf{r}^{\prime}\right|^{2}+s^{2}\right)^{1 / 2}$ for softening parameter $s$. In order to treat the force on the star, the mass in each cell is treated as a point mass at the cell's center, with the same softening parameter used for the star. The star's position is integrated from step $i$ to $i+1$ by the following: $\mathbf{v}^{i}=\mathbf{v}^{i-1 / 2}+0.5 \mathbf{a}^{i} \Delta t^{i-1}, \mathbf{v}^{i+1 / 2}=\mathbf{v}^{i}+0.5 \mathbf{a}^{i} \Delta t^{i}$, and $\mathbf{x}^{i+1}=\mathbf{x}^{i}+\mathbf{v}^{i+1 / 2} \Delta t^{i}$. This algorithm is sufficient for maintaining, on average, the system's center of mass at the grid center.

Each disk is evolved on an $r, \phi, z=256,512,64$ grid, with a spatial resolution of $\Delta r, r \Delta \phi$, and $\Delta z=2,2 \pi r / 512,2$ AU. Mirror symmetry is assumed about the midplane, and the outer grid boundaries are outflow boundaries. There is an outflow boundary near the star, but negligible mass passes through it in these simulations. Mass is added to the grid near the top of the $z$ outflow boundary between $r=60$ and 300 AU. The added mass is given a density profile of $\rho \sim r^{-p}$, a specific angular momentum $\left(G M_{\text {star }} r^{3} /\left(r^{2}+s^{2}\right)\right)^{1 / 2}$, an initial $v_{r}=0$, and an initial $v_{z}=-\left(2 G M_{\text {star }} / r\right)^{1 / 2}$. Upon reaching a disk mass of $0.1 M_{\odot}$, a random density perturbation is imposed with a maximum variation of $\pm 10 \%$. For all simulations, the disk is stable against GIs when the noise is added. For two of the simulations (see below) a softening of $s=20$ AU was applied. This softening causes an error in the epicyclic frequency of just under $10 \%$ at $r \sim 50 \mathrm{AU}$ when compared with the Keplerian frequency and $3 \%$ at $r \sim 100 \mathrm{AU}$.

\section{SIMULATIONS AND RESULTS}

Four simulations (SIMA, SIMB, SIMC, and SIMD) are shown in Figure 1. All disks except for SIMC fragment, and evolve isothermally except in very high-density regions. Because the vertically integrated midplane $\tau$ never becomes larger than a few, except in clumps, the above radiative transfer approximation is reasonable. Clumps reach temperatures in excess of $100 \mathrm{~K}$.

1. SIMA: the protostar is set to $0.3 M_{\odot}$. The initial mass loading $\dot{M}_{d} \sim 10^{-4} M_{\odot} \mathrm{yr}^{-1}$ until the disk mass $M_{d}=$ $0.1 M_{\odot}$, after which $\dot{M}_{d}$ is reduced to $10^{-5} M_{\odot} \mathrm{yr}^{-1}$. The infall density profile is set to $p=1.5$. A softening $s=20 \mathrm{AU}$ is used because the accretion rate is less than what is used in the other simulations, requiring a longer evolution. The simulation is evolved for $16,400 \mathrm{yr}$ (3 $P_{200}$, orbital periods at $r \sim 200 \mathrm{AU}$ ), and reaches a disk mass of $0.21 M_{\odot}$ before the simulation is stopped. Dense spiral waves develop, and a condensation forms near $r \sim 70$ AU. Fragmentation appears to be near wave 

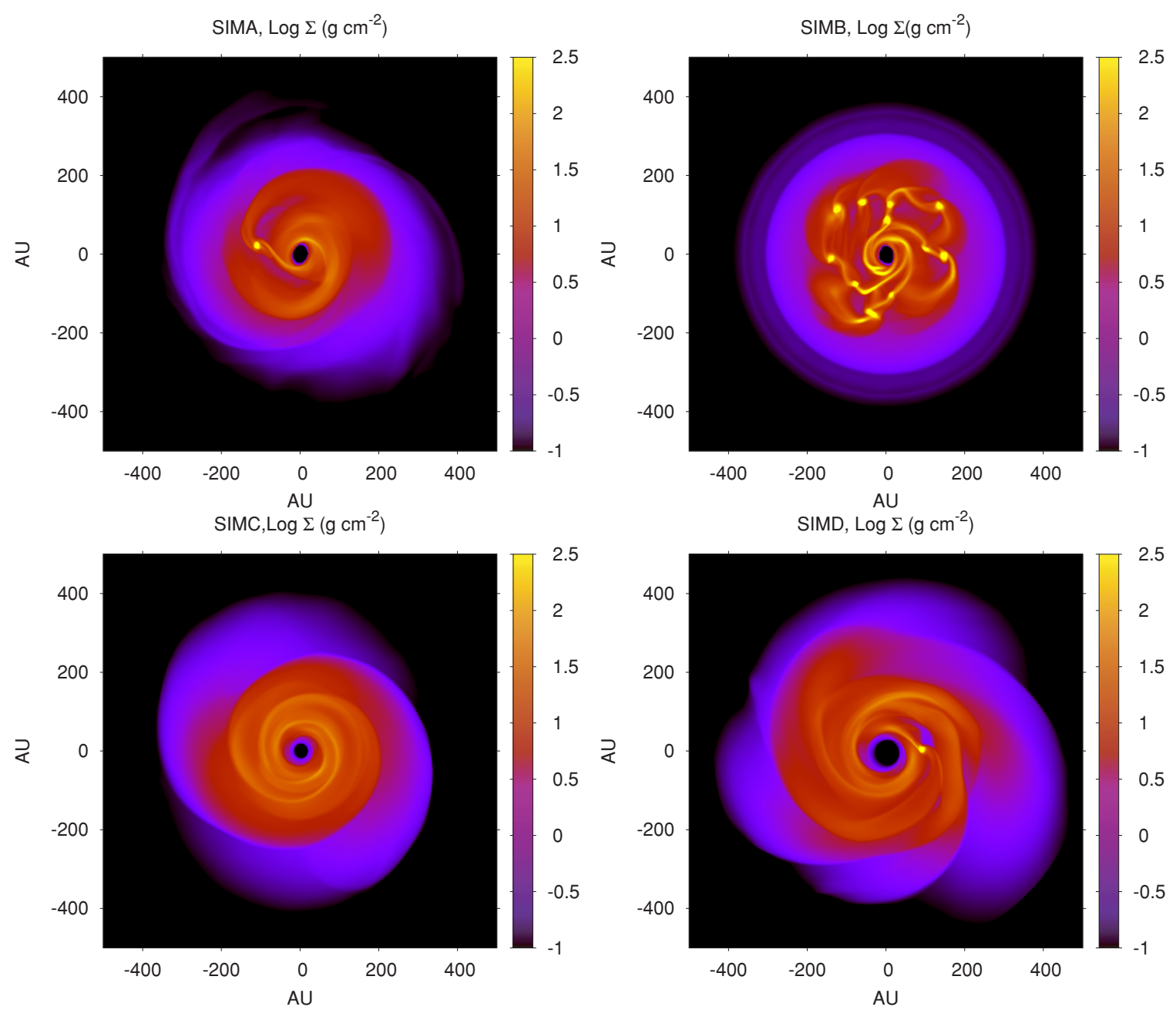

Figure 1. Surface density snapshots at the end of each simulation. Strong bursts of GI activity occur in each disk. For three of the simulations, these bursts lead to fragmentation and clump formation. Movies of these simulations can be viewed at http://www.aaroncboley.net under the Movies tab.

corotation, in agreement with Durisen et al. (2008). By the end of the simulation, the clump has grown to $20 M_{J}$ and is located at $r \sim 110$ AU. As a cautionary check, a portion of this simulation was rerun with CHYMERA's normal radiative transfer algorithm. The disk behaved isothermally.

2. $S I M B$ : the protostar is set to $1 M_{\odot}$, and $\dot{M}_{d} \sim 10^{-4} M_{\odot} \mathrm{yr}^{-1}$ for the duration of the simulation. The simulation is evolved for $5000 \mathrm{yr}\left(\sim 1.8 P_{200}\right)$, and the disk grows to $0.52 M_{\odot}$. The infall density profile $p=1.5$, and no softening is applied. Mass loading drives $Q$ below unity outside $r \sim 100$ $\mathrm{AU}$, and the disk fragments into 11 condensations by the time the simulation is stopped, ranging in mass from $\sim 4$ to $14 M_{J}$.

3. SIMC: similar to SIMB, but the mass accretion is halted when $M_{d}=0.33 M_{\odot}$. At this mass, $Q$ in a region near $r \sim 90$ AU drops below unity. This simulation investigates whether the disk can recover from the initial mass loading and avoid fragmentation. When GIs did set in, they rapidly transported mass both away and toward the star, so a softening of $s=20 \mathrm{AU}$ was applied and the inner disk boundary was moved from $r \sim 20$ AU to $r \sim 6$ AU. The initial simulation without softening shows a similar behavior, but was not evolved beyond the initial burst of GIs. The burst transports mass efficiently and $Q$ reaches a mass-weighted average $Q \sim 1.3$ between $r \sim 100$ and
$200 \mathrm{AU}$. This simulation is evolved for $9200 \mathrm{yr}\left(\sim 3.3 P_{200}\right)$. Although a longer integration may be required to ascertain whether the disk will eventually fragment, the simulation does indicate that fragmentation is not guaranteed in the outer disk.

4. SIMD: the protostar is set to $1 M_{\odot}$, and the mass accretion rate is $10^{-4} M_{\odot} \mathrm{yr}^{-1}$. As in SIMC, the mass accretion is halted once $M_{d}=0.33 M_{\odot}$. The density profile for the infalling material $p=0.5$. This choice for $p$ places the minimum $Q$ further out in the disk, such that $Q$ first drops below unity near $r \sim 140$ AU. Unlike SIMC, the spiral waves are unable to redistribute the mass enough to avoid fragmentation. The disk forms a $6 M_{J}$ clump at $r \sim 140 \mathrm{AU}$, which is subsequently transported to just inside $r \sim 100 \mathrm{AU}$ and grows to $11 M_{J}$ by the end of the simulation. The disk is evolved for $8600 \mathrm{yr}\left(\sim 3 P_{200}\right)$.

Wide semimajor axis, $a$, gas giants (WaGGs) and at least one brown dwarf (BD) are formed in these simulations. All disks go through strong burst-like phases, consistent with the arguments in Section 1. Although a high $\dot{M}$ is used for SIMB, SIMC, and SIMD, disk fragmentation is still expected for $\dot{M} \sim 10^{-5} M_{\odot} \mathrm{yr}^{-1}$, as seen in SIMA. The local Jeans length is resolved by at least four cells throughout the simulations (Truelove et al. 1997; Nelson 2006), where the local cell size is taken to be the geometric mean of the cell dimensions. These 
results indicate that fragmentation at large disk radii should be common and that WaGGs can be explained by in situ formation. These simulations demonstrate that the number or fragments and the fragment masses depend on how the disk is assembled. Studies investigating the clump mass spectrum of extended, massive protoplanetary disks, e.g., SW2009, are very sensitive to initial conditions.

Although the fate of these clumps is unknown, the results of Mayer et al. (2004) and SW2009 indicate that some condensations can survive. Radiative feedback, which is neglected in the current radiative cooling algorithm, should limit clump growth. Detailed simulations that can resolve the photosphere of a fragment are required to address this concern, which is beyond the resolution limit in these simulations.

These disks are evolved through only one burst. Vorobyov \& Basu (2005; hereafter VB2005) found in their two-dimensional simulations that several bursts can occur during the disk accretion phase. Whether a system forms $\mathrm{WaGGs/BDs}$ and retains them may be dependent on the details of the last GI burst. Because these simulations suggest that disks should be susceptible to fragmentation during their formation, they also support the possibility of a clump-driven outburst mechanism for FU Orionis objects (VB2005). The FU Orionis phenomenon is characterized by a rapid increase in the optical brightness of a young $\mathrm{T}$ Tauri object, typically 5 magnitudes over a few to tens of years. Accretion from the inner disk onto the star is estimated to be as high as $10^{-4} M_{\odot} \mathrm{yr}^{-1}$ (Hartmann \& Kenyon 1996). Because FU Ori objects have decay timescales of $\sim 100 \mathrm{yr}$, an entire minimum mass solar nebula $\left(\sim 0.01 M_{\odot}\right)$ can be accreted onto the protostar during an event. The best explanation for the optical outburst is a thermal instability (TI) (Bell \& Lin 1994). However, prodigious mass flux through $r \sim 0.1 \mathrm{AU}$ seems to be required to drive the TI. A tidally-disrupted WaGG could supply the inner disk with $0.01 M_{\odot}$ mass, even when only the extended disk is gravitationally unstable. Helled et al. (2006) calculated the contraction time for a Jupiter-mass clump, and found that such a clump takes $3 \times 10^{5} \mathrm{yr}$ to reach a central temperature of $2000 \mathrm{~K}$, i.e., the temperature required to dissociate enough $\mathrm{H}_{2}$ to lead to rapid collapse. This suggests that a $\mathrm{W} a \mathrm{GG}$ has sufficient time to be transported inside $r \sim 1$ AU before it reaches mean densities that are high enough to avoid tidal disruption, where the disruption radius $r_{t} \sim 8 \times 10^{-3}\left(\bar{\rho} / \mathrm{g} \mathrm{cm}^{-3}\right)^{-1 / 3}\left(M / M_{\odot}\right)^{1 / 3}$ AU. The Helled et al. model remains at a mean density of $\bar{\rho} \sim 2 \times 10^{-7} \mathrm{~g} \mathrm{~cm}^{-3}$ for $\sim 10^{5} \mathrm{yr}$. This density corresponds to a disruption radius $r_{t} \sim 1 \mathrm{AU}$ for a $0.3 M_{\odot}$ star. An immediate observational signature of this mechanism would be a large shift in the radial velocity of the protostar just before an outburst.

\section{THE TWO MODES OF PLANET FORMATION}

The Boss (1997) advancement of the disk instability mechanism has spawned a decade's worth of work examining GIs as a formation mechanism for gas giant planets (see Durisen et al. 2007's review). One of the principal reasons for the mechanism remaining contested for so long is that, like core accretion, the mechanism works under the right conditions. Rafikov (2005, 2007), Boley et al. (2006, 2007a), Stamatellos \& Whitworth 2008, and Boley \& Durisen (2008) argue that radiative cooling timescales are too long for fragmentation out to $r \sim 40 \mathrm{AU}$ and that sustained convection does not cause fragmentation. This is also consistent with the Nelson et al. (2000) two-dimensional radiative hydrodynamics simulations, who assumed a polytropic vertical density structure in order to calculate a photosphere temperature. Convection pushes the entropy gradient toward zero, so a vertical polytropic density stratification assumes efficient convection. Observationally, the planet-metallicity relationship (Valenti \& Fischer 2005) indicates that planet formation favors a high-metallicity environment, which is strong evidence for core accretion (cf. Boss 2005). The estimated core masses of Jupiter and Saturn (Saumon \& Guillot 2004; Militzer et al. 2008), along with the ice giants, support the core accretion mechanism. This evidence suggests that the dominant formation mechanism for gas giants inside $r \sim 100 \mathrm{AU}$ is core accretion.

In contrast to the conditions inside $r \sim 100 \mathrm{AU}$, optical depths should approach unity for a substantial $\Delta r$ in the extended disk, as occurs in these simulations. Efficient radiative cooling, long orbital periods, and an equilibrium O:P ratio combine to favor fragmentation in the extended disk. WaGG formation as a result of mass loading represents the first mode of planet formation, and takes place in the first $10^{5} \mathrm{yr}$ of the disk's lifetime. Core accretion, which can continue after the main disk formation phase, represents the slower, second mode of gas giant formation. The survival of $\mathrm{WaGG}$ will likely depend on the disk and mass accretion conditions during the last burst of GI activity.

Both core accretion and disk instability are able to form gas giants under suitable conditions. In the inner disk, conditions favor core accretion, and in the extended disk, conditions favor disk instability. Scattering and planet-disk interactions should wash out any strict desert between the two formation regimes, but a bimodal population of gas giant planet semimajor axes should still be present. Because core accretion is expected to be less efficient in low-metallicity systems, the ratio of $\mathrm{WaGGs}$ to planets at small $r$ should increase with decreasing metallicity.

I thank the referee for pointing out a complementary study by Rafikov (2009), which was posted during the review of this manuscript. Rafikov's work discusses fragmentation conditions and the stability of disks against high $\dot{M}$, while the study presented here demonstrates that a disk can be pushed realistically toward fragmentation.

I thank R. H. Durisen, L. Mayer, G. Lake, R. Teyssier, O. Agertz, D. Stamatellos, and the referee for comments that improved this manuscript. This research was supported by a Swiss Federal Grant and the University of Zurich. The presented simulations were run on NASA Advanced Supercomputing facilities.

\section{REFERENCES}

Bell, K. R., \& Lin, D. N. C. 1994, ApJ, 427, 987

Boley, A. C. 2007, PhD Thesis, Indiana Univ.

Boley, A. C., \& Durisen, R. H. 2006, ApJ, 641, 534

Boley, A. C., \& Durisen, R. H. 2008, ApJ, 685, 1193

Boley, A. C., Durisen, R. H., Nordlund, Å, \& Lord, J. 2007a, ApJ, 665, 1254

Boley, A. C., Hartquist, T. W., Durisen, R. H., \& Michael, S. 2007b, ApJ, 656, L89

Boley, A. C., Mejía, A. C., Durisen, R. H., Cai, K., Pickett, M. K., \& D’Alessio, P. 2006, ApJ, 651, 517

Boss, A. P. 1997, Science, 276, 1836

Boss, A. P. 1998, ApJ, 503, 923

Boss, A. P. 2004, ApJ, 610, 456

Boss, A. P. 2005, ApJ, 629, 535

Boss, A. P. 2006, ApJ, 637, L137

Boss, A. P. 2008, ApJ, 677, 607

Cai, K., Durisen, R. H., Michael, S., Boley, A. C., Mejía, A. C., Pickett, M. K., \& D’Alessio, P. 2006, ApJ, 636, L149

Cameron, A. G. W. 1978, Moon Planets, 18, 5

D’Alessio, P., Calvet, N., \& Hartmann, L. 2001, ApJ, 553, 321 
Durisen, R. H., Boss, A., Mayer, L., Nelson, A., Quinn, T., \& Rice, K. 2007, in Protostars and Planets V, ed. B. Reipurth, D. Jewitt, \& K. Keil (Tucson, AZ: Univ. Arizona Press), 607

Durisen, R. H., Hartquist, T. W., \& Pickett, M. K. 2008, Ap\&SS, 317, 3D

Gammie, C. F. 2001, ApJ, 553, 174

Goodman, J. 2003, MNRAS, 339, 937

Hartmann, L., \& Kenyon, S. J. 1996, ARA\&A, 34, 207

Helled, R., Podolak, M., \& Kovetz, A. 2006, Icarus, 185, 64

Kalas, P., et al. 2008, Science, 322, 1345

Lafreniere, D., Jayawardhana, R., \& van Kerkwijk, M. H. 2008, ApJ, 689, L153

Lodato, G., \& Rice, W. K. M. 2004, MNRAS, 351, 630

Luhman, K. L., et al. 2006, ApJ, 649, L894

Matzner, C. D., \& Levin, Y. 2005, ApJ, 628, 817

Mayer, L., Lufkin, G., Quinn, T., \& Wadsley, J. 2002, Science, 298, 1756

Mayer, L., Lufkin, G., Quinn, T., \& Wadsley, J. 2007, ApJ, 661, 77

Mayer, L., Quinn, T., Wadsley, J., \& Stadel, J. 2004, ApJ, 609, 1045

Mejía, A. C., Durisen, R. H., Pickett, M. K., \& Cai, K. 2005, ApJ, 619, 1098

Militzer, B., Hubbard, W. B., Vorberger, J., Tamblyn, I., \& Bonev, S. A. 2008, ApJ, 688, L45

Nelson, A. F. 2006, MNRAS, 373, 1039
Nelson, A. F., Benz, W., Adams, F. C., \& Arnett, D. 1998, ApJ, 502, 342

Nelson, A. F., Benz, W., \& Ruzmaikina, T. V. 2000, ApJ, 529, 357

Pickett, B. K., Mejía, A. C., Durisen, R. H., Cassen, P. M., Berry, D. K., \& Link, R. P. 2003, ApJ, 590, 1060

Pollack, J. B., Hubickyj, O., Bodenheimer, P., Lissauer, J. J., Podolak, M., \& Greenzweig, Y. 1996, Icarus, 124, 63

Rafikov, R. R. 2005, ApJ, 621, L69

Rafikov, R. R. 2007, ApJ, 662, 642

Rafikov, R. R. 2009, arXiv:0901.4739

Rice, W. K. M, Lodato, G., \& Armitage, P. J. 2005, MNRAS, 364, L56

Saumon, D., \& Guillot, T. 2004, ApJ, 609, 1170

Shakura, N. I., \& Syunyaev, R. A. 1973, A\&A, 24, 337

Stamatellos, D., Hubber, D. A., \& Whitworth, A. P. 2007, MNRAS, 382, L30

Stamatellos, D., \& Whitworth, A. P. 2008, A\&A, 480, 879

Stamatellos, D., \& Whitworth, A. P. 2009, MNRAS, 392, 413

Tomley, L., Steiman-Cameron, T. Y., \& Cassen, P. 1994, ApJ, 422, 850

Toomre, A. 1964, ApJ, 139, 1217

Truelove, J. K., Klein, R. I., McKee, C. F., Holliman, J. H., II, Howell, L. H., \& Greenough, J. A. 1997, ApJ, 489, L179

Valenti, J. A., \& Fischer, D. A. 2005, ApJS, 159, 141

Vorobyov, E. I., \& Basu, S. 2005, ApJ, 633, 137 